\title{
ALFABETIZAÇÃO INICIAL VIA ENSINO SISTEMÁTICO PARA CRIANÇAS COM COMPORTAMENTOS EXTERNALIZANTES
}

\author{
Priscila Meireles Guidugli ${ }^{1} \mathbb{D}$; Ana Claudia Moreira Almeida-Verdu ${ }^{1}(\mathbb{D}$
}

\section{RESUMO}

Comportamentos externalizantes como birra e agressividade são frequentemente concorrentes à aprendizagem acadêmica. A maior frequência de um comportamento em detrimento de outro pode indicar falhas no planejamento das condições de ensino. Verificou-se se três meninos, entre nove e 10 anos, com comportamentos externalizantes aferidos pelo instrumento TRF e não alfabetizados, aprenderiam leitura e escrita quando expostos ao ALEPP (Aprendendo a Ler e Escrever em Pequenos Passos). Programa de ensino com instrução baseada em equivalência e abordagem operacional de comportamentos simbólicos, com quatro unidades, divididas em 17 passos de ensino, precedidos e sucedidos por testes. Não houve intervenção sobre comportamentos externalizantes. Os resultados mostraram mais de $80 \%$ de acertos em leitura e escrita. Futuras pesquisas devem verificar a replicabilidade desses resultados com mais participantes e se a aquisição de repertórios acadêmicos afetaria a frequência de comportamentos externalizantes, considerando as habilidades do professor de reforçar repertórios acadêmicos em detrimento dos comportamentos externalizantes.

Palavras-chave: ensino; aprendizagem escolar; problemas de comportamento.

\section{Initial literacy via systematic teaching for children with externalizing behaviors}

\section{ABSTRACT}

Externalizing behaviors such as tantrum and aggression are often competing with academic learning. The higher frequency of one behavior over another may indicate flaws in the planning of teaching conditions. It was verified if three boys, between nine and 10 years old, with externalizing behaviors measured by the TRF instrument and not literate, would learn to read and write when exposed to ALEPP (Learning to Read and Write in Small Steps). Teaching program with instruction based on equivalence and operational approach to symbolic behaviors, with four units, divided into 17 teaching steps, preceded and succeeded by tests. There was no intervention on externalizing behaviors. The results showed more than $80 \%$ of correct answers in reading and writing. Future research should verify the replicability of these results with more participants and if the acquisition of academic repertoires would affect the frequency of externalizing behaviors, considering the teacher's abilities to reinforce academic repertoires at the expense of externalizing behaviors.

Keywords: teaching; school learning; behavior problems.

\section{Alfabetización inicial vía enseñanza sistemática para niños con comportamientos externalizantes}

\section{RESUMEN}

Comportamientos externalizantes como rabietas y agresividad, son frecuentemente competidores al aprendizaje académica. La mayor frecuencia de un comportamiento en detrimento de otro puede indicar fallas en la planificación de las condiciones de enseñanza. Se verificó si tres niños, entre nueve y 10 años, con comportamientos externalizantes evaluados por el instrumento TRF y no alfabetizados, aprenderían lectura y escritura cuando expuestos al ALEPP (Aprendiendo y Leer y Escribir en Pequeños Pasos). Programa de enseñanza con instrucción basada en equivalencia y abordaje operacional de comportamientos simbólicos, con cuatro unidades, divididas en 17 pasos de enseñanza, precedidos y sucedidos por testes. No hubo intervención sobre comportamientos externalizantes. Los resultados apuntaron más del $80 \%$ de aciertos en lectura y escritura. Futuras investigaciones deben verificar la replicabilidad de esos resultados con más participantes y si la adquisición de repertorios académicos evaluaría la frecuencia de comportamientos externalizantes, considerando las habilidades del profesor de reforzar repertorios académicos en detrimento de los comportamientos externalizantes.

Palabras clave: enseñanza; aprendizaje escolar; problemas de comportamiento.

1 Universidade Estadual Paulista Júlio de Mesquita Filho - Bauru - SP - Brasil; pity_mg@yahoo.com.br; ana.verdu@unesp.br 


\section{INTRODUÇÃO}

Para a Análise do Comportamento, leitura e escrita são processos comportamentais complexos, passíveis de análise e ensino de forma eficaz a partir da sua descrição e operacionalização, isto é, descrição da resposta alvo e das condições sob as quais ocorrem. $\mathrm{O}$ atendimento à diversidade de alunos com atrasos na aprendizagem de leitura e escrita tem sido o alvo de muitos pesquisadores que buscam descrever procedimentos de ensino eficazes no ensino dessas habilidades (de Rose, 2005).

Um dos modelos adotados tem sido o das relações de equivalência, um modelo operacional de comportamento simbólico (Sidman \& Tailby, 1982; Sidman, 2000). O modelo consiste no estabelecimento de pelo menos duas discriminações condicionais do tipo "se... então..." com um elemento em comum. O procedimento mais comumente empregado é o MTS (matching to sample) que consiste na apresentação de um estímulo com a função de modelo e pelo menos dois com a função de comparação, cuja tarefa do aprendiz é selecionar um dos estímulos de comparação, condicionalmente ao modelo apresentado (de Rose, de Souza, Rossito, \& de Rose, 1989).

A título de exemplo, se uma criança ouve "boneca" e aponta a própria boneca em detrimento de outros objetos e, se diante da palavra "boneca" ela aponta a palavra escrita BONECA em detrimento de outras palavras, a criança pode, sem ensino direto, relacionar a palavra escrita ao objeto e vice-versa, estabelecendo relações entre estímulos fisicamente diferentes (Sidman \& Taiby, 1982), sendo esta uma medida operacional do comportamento simbólico e da compreensão leitora. Considerando que a palavra ditada, a palavra escrita e a figura são permutáveis entre si e compõem uma classe de estímulos equivalentes, se a criança já é capaz de dizer "boneca" diante do objeto, as relações de controle exercidas pelo objeto "boneca" podem ser estendidas para a palavra escrita BONECA, e a criança pode passar a dizer "boneca" diante da palavra escrita, de tal forma que todos os estímulos da classe passam a controlar a resposta de dizer "boneca" (Sidman, 2000), sendo essa uma medida adicional da leitura com compreensão.

O modelo das relações de equivalência entre estímulos (Sidman \& Taiby, 1982) e entre estímulos e respostas (Sidman, 2000) tem sido a base de programas de ensino de leitura generalizada (Zaine, Domeniconi, \& de Rose, 2014). Considerando as relações entre estímulos e respostas, se o repertório de construção da resposta for estabelecido, por exemplo (B-O-N-E-C-A), um outro processo, o de generalização recombinativa, pode fazer emergir novas palavras a partir de recombinações de unidades menores das palavras ensinadas (BOCA, BONÉ, CABO). Um dos procedimentos que favorece a generalização recombinativa é o Constructed Response Matching to Sample (CRMTS), (de Souza et al., 2009).
O estudo da aplicação desse modelo tem demonstrado resultados promissores no ensino de leitura e escrita e seus pré-requisitos em indivíduos com diferentes necessidades especiais de ensino, tais como, Transtorno do Espectro Autista (Gomes \& de Souza, 2016), escolares com desenvolvimento típico, mas com reconhecidos atrasos na aprendizagem da leitura (Reis, de Souza, \& de Rose, 2009), crianças com deficiência intelectual (Benitez \& Domeniconi, 2012), com deficiência auditiva usuários de LIBRAS (Santos \& Almeida-Verdu, 2012), crianças com deficiência visual (Quinteiro, 2015) e com deficiência auditiva e implante coclear (Lucchesi, Almeida-Verdu, Buffa, \& Bevilacqua, 2015). Em larga escala, o ALEPP tem sido testado em sala de recursos (Cravo \& Almeida-Verdu, 2018) e em escala maior ainda, em parceria com a Secretaria de Educação de um dos municípios da região Metropolitana de São Paulo, avaliando a eficácia do programa com mais de 500 alunos com dificuldades na aprendizagem de leitura e escrita em alunos de 20 a 5 을 ano do ensino fundamental com idades entre 7 e 15 anos, de 8 escolas municipais (de Souza et al., 2019), ambos com resultados positivos considerando o comportamento alvo que é a aprendizagem de leitura e escrita.

Nas pesquisas realizadas, embora, de forma geral, os participantes tenham um bom desempenho em tarefas de seleção de palavras ou de figuras condicionalmente às respectivas palavras ditadas, nos pré-testes, nas tarefas de leitura e escrita sob ditado o desempenho não é satisfatório. Após passarem pelos programas de ensino, as porcentagens de acertos de leitura e de escrita aumentam consideravelmente, chegando até a $100 \%$ de acertos nas palavras de treino e um aumento considerável em palavras novas, com e sem sentido. A leitura recombinativa representa ampliação do repertório do aluno, que passa a ler palavras não ensinadas diretamente, como consequência de aprendizagens prévias. Quando o aluno começa a apresentar leitura recombinativa, pode-se considerar que ele está, efetivamente, aprendendo a ler fluentemente.

O software Aprendendo a Ler e Escrever em Pequenos Passos - ALEPP (Rosa Filho, de Rose, Souza, Hanna, \& Fonseca, 1998) objetiva promover a aprendizagem de repertórios essenciais à leitura e escrita, e foi desenvolvido com base na literatura sobre relações de equivalência. Suas principais características são: procedimentos de ensino de discriminações condicionais entre palavras e sílabas ditadas e palavras e sílabas impressas que minimizam erros, consequências diferenciais para respostas de acerto e de erro e a progressão gradual do conteúdo a ser ensinado à medida que o aprendiz vai obtendo precisão em repertórios dos mais simples para os mais complexos. Inclui módulos de ensino que já se mostraram eficazes em pesquisas de laboratório e, atualmente, são disponibilizados para uso em diferentes 
condições clínicas e educacionais, permitindo o seu uso e verificação da efetividade em situações mais aplicadas e em larga escala. ${ }^{1}$

Este estudo questionou a efetividade do ALEPP em crianças com comportamentos externalizantes, que são marcados por impulsividade, agressão, agitação, características desafiantes e antissociais (Achenbach \& Rescorla, 2000). Esses comportamentos são dirigidos ao ambiente físico (i.e., jogar objetos no chão, quebrar coisas) ou social (i.e., agredir alguém física ou verbalmente). Bandeira, Rocha, Souza, Del Prette e Del Prette (2006) apontam que na literatura da Psicologia Comportamental tem sido observada a co-ocorrência entre comportamentos problema e dificuldades de aprendizagem, atingindo em escala internacional cerca de $12 \%$ das crianças em idade escolar. No entanto, ainda é controversa a origem e direção causal da relação entre as duas variáveis. Esse trabalho considerou que, se são comportamentos concorrentes, a escolha da emissão de comportamentos acadêmicos em detrimento de comportamento externalizante pode ser afetada pelas consequências reforçadoras disponíveis (Souza \& Coelho, 2019). Consequências imediatas, contínuas, mas diferenciadas para acertos e erros, favorecendo acertos e minimizando erros planejadas no ALEPP podem favorecer a emissão de comportamentos de leitura e escrita requeridos pelo ALEPP, em detrimento dos externalizantes.

Dessa maneira, esse trabalho propõe que tecnologias de ensino baseadas em treinos bem estruturados, sequenciados em pequenos passos, com a apresentação de poucos estímulos por tentativa e com aplicação sistemática, imediata e consistente de esquemas de reforçamentos diferenciais para a resposta atencional possam minimizar prejuízos decorrentes de déficits atencionais (Duarte $\&$ de Rose, 2006). No entanto, a literatura que relaciona problemas de comportamento e efeitos de programas de intervenção em leitura e escrita ainda é escassa (D'abreu \& Marturano, 2010) por um lado. Por outro, a Psicologia e a Educação têm acumulado um debate ao longo de décadas sobre essa questão identificando condições de desigualdade entre os aprendizes, que promovem a exclusão de uma parcela com características estigmatizantes, e que procura identificá-las e neutralizá-las passando por variáveis mais distais como contextos comunitários e formação de grêmios e associações (i.e., Guzzo, Mezzalira, Moreira, Tizzei, \& Silva Neto, 2010). Para além de propor soluções técnicas para problemas supostamente centrados no indivíduo, esse trabalho alinha-se com esse debate que analisa as variáveis responsáveis pela não aprendizagem de conteúdos escolares por determinados grupos de

${ }^{1}$ O programa é disponibilizado pela plataforma GEIC (Gerenciador de Ensino Individualizado por Computador), sediada na UFSCar. estudantes (i.e., Paula \& Tfouni, 2009) reconhecendo que condições externas ao aprendiz podem favorecer ou dificultar a aprendizagem, tanto de comportamentos acadêmicos como os denominados externalizantes. No entanto, esse trabalho propõe a análise e a intervenção sobre variáveis mais proximais ao comportamento alvo, neste caso, aspectos procedimentais relacionados à aprendizagem de leitura e escrita. Dessa forma, será pelo planejamento de condições de ensino que um comportamento pode ser mais favorecido em detrimento de outro.

Considerando a co-ocorrência de déficits acadêmicos e comportamentos externalizantes (Bandeira, et al. 2006), ainda que a literatura mais recente seja escassa, foram encontrados registros anteriores de recomendações de intervenções tais como atuar na redução dos problemas de comportamento seguida da otimização de habilidades acadêmicas (Harris \& Sherman, 1974).

Uma direção inversa a essa seria intervir primeiramente sobre o desempenho acadêmico do aluno, com respostas diretamente relacionadas com a realização das tarefas e verificar os efeitos sobre os denominados comportamentos problema. Guilhardi, Betini e Camargo (1977) adotaram essa proposta com um participante de sete anos, que tinha dificuldades em leitura e escrita e apresentava problemas de comportamento (levantar-se, andar pela classe, brigar com os colegas, empurrar as carteiras). A intervenção consistiu em reforçar apenas o desempenho acadêmico. Como resultados constataram que foi possível reduzir a frequência dos comportamentos externalizantes. Esse estudo demonstrou que o comportamento acadêmico pode ser alvo de ensino direto sem que se tenha que eliminar, antes, os comportamentos externalizantes concorrentes às tarefas acadêmicas.

Esses resultados fortalecem a hipótese de que problemas de comportamento e problemas de aprendizagem, no caso do aluno do estudo, estavam sob esquema de reforçamento concorrente e eram incompatíveis. Quando as contingências aumentaram o valor reforçador para a emissão de comportamentos acadêmicos em detrimento dos incompatíveis com esses, o aluno passou a apresentar, com frequência maior, os comportamentos relacionados à tarefa acadêmica.

É fato que uma análise funcional dos problemas de comportamento poderia revelar o que os mantém (e.g., se o aluno somente recebe atenção, seja ela um elogio ou uma bronca, mediante tais comportamentos; ou se emite tais comportamentos como forma de fuga/ esquiva de situações aversivas com práticas de pessoas da escola ou com a própria situação de aprendizagem). Uma intervenção que promovesse a alteração das contingências de reforço presentes durante a emissão dos problemas de comportamento poderia alterar sua probabilidade de ocorrência naqueles contextos (Han- 
ley, Iwatta, \& McCord, 2003). No entanto, este não é o escopo desse estudo. $O$ presente estudo propõe que a intervenção sobre o repertório acadêmico embora não prescinda de uma intervenção sobre o repertório social pode não depender que ela ocorra, quando os problemas de comportamento externalizantes são concorrentes.

Dessa forma, a intervenção avaliou se as crianças com comportamentos externalizantes e não alfabetizadas, ao receberem ensino sistemático em leitura e escrita do software ALEPP, aprenderiam as respostas acadêmicas ensinadas e diminuiriam a frequência de comportamentos externalizantes monitorados pelo Teacher Report Form (TRF).

\section{Participantes}

\section{MÉTODO}

Participaram da pesquisa três meninos com idades entre 9 e 10 anos, frequentando o 3ㅇ ou 4 ano do Ensino Fundamental I, com problemas de comportamentos externalizantes e não alfabetizados. Na etapa de triagem e seleção dos participantes, os professores indicavam alunos com problemas de comportamento e dificuldades de aprendizagem e responderam ao Teacher Report Form (TRF), melhor descrito na seção de "Ambiente, Materiais e Instrumentos", instrumento de rastreamento de problemas de comportamento externalizantes, internalizantes e déficits atencionais; para essa pesquisa foram considerados apenas os resultados acerca dos problemas de comportamentos externalizantes.

As crianças eram avaliadas pelo Diagnóstico de Leitura e Escrita (DLE), também sediado no servidor GEIC e precedia o Programa ALEPP, mais bem descrito na seção de "Ambiente, Materiais e Instrumentos e que aferiu a porcentagem de acertos em relações entre estímulos e entre estímulos e respostas de leitura oral e escrita. A Tabela 1 demonstra o resultado da triagem e as principais características dos participantes.

A Pesquisa foi aprovada no Comitê de Ética em Pesquisa da Faculdade de Ciências da Unesp Bauru,
Parecer $\mathrm{n}$ - 254.407) e todos os procedimentos éticos foram adotados.

\section{Ambiente, Materiais e Instrumentos}

A coleta foi realizada em uma sala disponibilizada pela escola onde os alunos recebiam ensino formal. A sala era multifuncional e era equipada com um microcomputador que gerenciava as atividades planejadas no ALEPP, caixas de som por onde o aluno ouvia as instruções e um mouse pelo qual ele realizava a tarefa. Permaneciam na sala o aluno e a experimentadora. Foram adotados os seguintes instrumentos:

Teacher Report Form - TRF (Achenbach \& Rescorla, 2000): investiga a frequência de 113 respostas indicativas de problemas de comportamento como "murmura ou faz barulhos estranhos na sala de aula?", "não consegue parar sentado, é inquieto ou hiperativo?", "é desobediente?" ou "mete-se em muitas brigas?" em escolares de 6 a 18 anos, respondidas pelo professor. Os dados são contabilizados em um software, o qual apresenta curvas, através de T Escores de 0 a 100, indicando se a criança apresenta problemas considerados clínicos (de 70 a 100), se está em uma área limítrofe (entre 66 e 69) ou não apresenta problemas em nível clínico (de 50 a 65), nas escalas externalizante e internalizante, apresentando ao final também uma análise global das escalas (total).

- O Diagnóstico de Leitura e Escrita (DLE), software incluído na plataforma GEIC, que avalia 16 tipos de relações que descrevem leitura e escrita, melhor descritas em "Delineamento e Procedimento". As relações exigiam diferentes tipos de respostas, nesse trabalho subdivididas em seleção, composição (escrita por seleção de sílabas na tela do computador) e produção oral (leitura).

Aprendendo a Ler e Escrever em Pequenos Passos - ALEPP (Rosa Filho et al., 1998): software disponibilizado pela plataforma GEIC, adotado para avaliação e ensino dos comportamentos de ler e escrever palavras, composto por uma sucessão de tarefas individuais, agrupadas em passos de ensino e esses em unidades. 0

Tabela 1. Caracterização dos Participantes Selecionados para a Pesquisa de Acordo com os Resultados da Triagem.

\begin{tabular}{|c|c|c|c|c|c|c|}
\hline \multirow{2}{*}{\multicolumn{2}{|c|}{ Idade }} & \multirow{2}{*}{ Série } & \multirow{2}{*}{ Diagnóstico prévio } & \multirow{2}{*}{ Externalizantes - TRF } & \multicolumn{2}{|c|}{ DLE (pré-teste) } \\
\hline & & & & & Leituraa $^{a}$ & Escrita $^{b}$ \\
\hline P1 & 10 & 3 & $\begin{array}{l}\text { F80.9 - Transtorno não especificado do } \\
\text { desenvolvimento da fala ou da linguagem }\end{array}$ & clínico & $56 \%$ & $87 \%$ \\
\hline P2 & 9 & 3응 & Nenhum & clínico & $0 \%$ & $60 \%$ \\
\hline P3 & 10 & 40 & Nenhum & clínico & $6,7 \%$ & $40 \%$ \\
\hline
\end{tabular}

Nota: De acordo com a Legenda $D L E$, ${ }^{a}$ Leitura é representado pela relação $C D$ e ${ }^{b}$ Escrita é representada pelas relações $A E$ e $A F$. Fonte: Elaboração própria. 
programa totaliza cinco unidades de ensino, subdividido em 20 passos; cada passo ensina três palavras por vez. A sessão durava em média 20 minutos (tempo médio para a execução de um passo) e ocorria três vezes por semana. O Quadro 1 exibe exemplos de tipos de tarefas e telas do programa considerando relações entre estímulos (tarefas baseadas em respostas de seleção) e entre estímulos e respostas (tarefas baseadas na composição e na produção oral).

\section{Delineamento e Procedimento}

O estudo foi dividido em três etapas.

Etapa 1 - As professoras responderam ao TRF para identificação e caracterização dos comportamentos externalizantes dos alunos indicados para o estudo. Em seguida, os participantes foram avaliados pelo Diagnós-

Quadro 1. Relações entre estímulos (tarefas baseadas em respostas de seleção) e entre estímulos e respostas (tarefas baseadas na composição e na produção oral), representações de telas e estímulos auditivos presentes no DLE e no ALEPP.

\begin{tabular}{|c|c|c|c|}
\hline Relações & Representação da Tela & Estímulo Auditivo & Tipo de Resposta \\
\hline Palavra ditada e figura & & “Aponte apito" & Seleção \\
\hline $\begin{array}{l}\text { Palavra ditada e } \\
\text { composição (ditado) }\end{array}$ & $\begin{array}{llllll}a & c & f & & & \\
a & e & b & u & & d\end{array}$ & "Escreva tatu" & Composição \\
\hline Figura e palavra escrita & & "Aponte a palavra" & Seleção \\
\hline $\begin{array}{l}\text { Palavra escrita e } \\
\text { composição (cópia) }\end{array}$ & 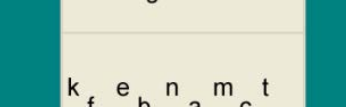 & "Escreva igual” & Composição \\
\hline $\begin{array}{l}\text { Palavra escrita e produção } \\
\text { oral (leitura) }\end{array}$ & bols & “O que está escrito?" & Produção oral \\
\hline
\end{tabular}

Fonte: Elaboração própria. 
tico de Leitura e Escrita (DLE), para caracterização do repertório em relações de leitura e de escrita sob ditado.

As tarefas do DLE eram organizadas em blocos de 15 tentativas, designadas por duas letras; a primeira representava as características do estímulo modelo (A - palavra ditada; B - figura; C - palavra escrita) e a segunda as características dos estímulos de comparação em tarefas de seleção ( $B$ - figura; $C$ - palavra escrita) ou da topografia da resposta em tarefas de composição ( $E$ - seleção de anagramas; F-manuscrita) e de produção oral ( $D$ - fala do participante). Não havia consequência programada para acerto e erro.

Nas tarefas de seleção, os participantes deveriam selecionar entre palavra impressa e figura, o estímulo de comparação correto condicional aos: estímulos visuais baseado em relações de similaridade física sendo figura-figura (BB) e palavra impressa-palavra impressa (CC); estímulos auditivos, palavra ditada-figura ( $A B)$ e palavra ditada-palavra impressa (AC); e estímulos visuais baseado em relações arbitrárias como as relações entre figura-palavra impressa (BC) e palavra impressa-figura (CB) (cf. exemplos do Quadro 1).

Nas tarefas de produção oral, após ser apresentado na tela do computador um estímulo (figura ou palavra impressa), os participantes deveriam ser capazes de emitir a resposta verbal designada como correta, realizando assim a nomeação da figura (BD), leitura de palavras (CD), letras (CD letr.), sílabas (CD síl.) e vogais (CD vog.) (cf. exemplos do Quadro 1).

Nas tarefas de composição os participantes deveriam produzir a resposta correta condicionada à: palavras ditadas pelo autofalante do computador, como nas tarefas de ditado por composição de letras (AE) ou manuscritas (AF) com papel e lápis; ou à palavras escritas como nas tarefas de cópia por composição de letras (CE) e manuscritas com papel e lápis (cf. exemplos do Quadro1).

Etapa 2 - Consistiu no ensino de leitura pela exposição às Unidades de Ensino do ALEPP, que incluem também pré e pós testes que verificam repertório de leitura e de ditado antes e depois de cada unidade de ensino como forma de verificar os efeitos deste.

Cada Unidade de Ensino era subdividida em passos e cada passo ensinava três palavras por vez; ao todo eram 20 passos de ensino totalizando 60 palavras. O critério de acertos para a conclusão de um passo e avançar para o próximo era de $100 \%$ de acertos; a conclusão de sucessivos passos de ensino estabeleceu uma rede de relações cumulativa entre palavra ditada, palavra escrita, figura e sílabas. Cada Unidade de Ensino era precedida e sucedida por testes de leitura baseada em seleção (BC e CB), de leitura oral (CD) e de ditado de palavras por composição a partir de sílabas e letras (AE). As avaliações antes e após cada unidade de ensino apresentavam não apenas as palavras ensinadas, mas também um conjunto de novas palavras compostas pela recombinação das sílabas das palavras ensinadas, para verificar se o aluno leria palavras novas, neste recorte serão considerados os resultados das palavras ensinadas.

Etapa 3 - As professoras responderam novamente ao TRF e os participantes foram expostos novamente ao Diagnóstico de Leitura e Escrita.

\section{RESULTADOS}

Nesta seção são apresentadas as porcentagens de acertos dos participantes nos pré e pós-testes gerais com as tarefas do Diagnóstico de Leitura e Escrita (DLE), as porcentagens de acertos nos pré e pós-testes de unidade do ALEPP e os resultados do TRF, medida de problema de comportamento a partir do relato do professor.

P1 foi exposto às cinco unidades do programa e os participantes $\mathrm{P} 2$ e $\mathrm{P} 3$ foram expostos somente às unidades de ensino 1 e 2 devido ao encerramento do ano letivo, início do período de férias escolares e mudança das crianças para uma escola com Ensino Fundamental II. Ainda que tenham sido expostos somente às duas unidades de ensino, o pós-teste de Diagnóstico de Leitura e Escrita (DLE) foi aplicado nos mesmos, pois concluíram o ensino de 10 passos de ensino, o que representa o ensino de 30 palavras.

\section{Diagnóstico de Leitura e Escrita - DLE}

As porcentagens de acertos obtidas pelos participantes nos pré (barras sólidas) e pós-testes (barras hachuradas) no DLE estão na Figura 1, agrupados de acordo com o tipo de resposta.

No Pré-teste, nas tarefas de seleção, todos os participantes obtiveram mais de $50 \%$ de acertos em todas as relações entre estímulos e, em algumas que envolvem relações de similaridade física entre figuras (BB), entre palavras escritas $(C C)$ e de reconhecimento da relação entre palavra ditada e figura $(A B)$, foram precisas, praticamente. Nas relações arbitrárias envolvendo palavra escrita, quais sejam, reconhecimento de palavras (AC), entre figura - palavra impressa (BC) e palavra impressa - figura (CB), P1 obteve acima de 93\% de acertos, P2 entre $53 \%$ e $73 \%$ e P3 entre $60 \%$ e $73 \%$. Nas tarefas de produção oral, a maior porcentagem de acertos foi de $53 \%$ obtida por P1 em leitura de palavras (CD); P2 não obteve acertos e P3 obteve apenas 7\% de acertos. Nas demais relações de produção oral, seja nomeação de figuras (BD), de letras $\left(\mathrm{CD}_{\text {letras }}\right)$ ou de sílabas $\left(\mathrm{CD}_{\text {sil }}\right)$, o desempenho foi superior a $50 \%$ de acertos. Nas relações que envolviam composição, os resultados em cópia (CE, por seleção de letras na tela e CF, manuscrita) sempre foram superiores aos de ditado (AE e AF), sendo os resultados mais baixos em ditado registrados para $\mathrm{P} 2 \mathrm{e}$ P3 (entre 60\% e 40\% de acertos).

Após a exposição às unidades de ensino pelo ALE- 
PP, os Pós-testes do DLE foram superiores para todas as relações envolvendo palavras impressas ( $A C, B C$ e $C B$ ), leitura de palavras (CD) ou escrita sob ditado (AE e $A F)$, sendo superiores a $85 \%$ de acertos ou chegando à precisão para os três participantes, tal como pode ser observado na Figura 1.

\section{Unidade de ensino do ALEPP}

As porcentagens de acertos dos Pré e Pós-teste das Unidades de Ensino estão apresentados na Figura 2.
Foram extraídos os dados das relações de nomeação de palavras $(C D)$, de ditado $(A E)$, e relações de seleção de figuras envolvendo palavras impressas e vice-versa (BC e CB).

As relações baseadas em seleção ( $B C$ e $C B$ ) que já eram boas, chegaram à precisão para todos os participantes. Embora P1 apresentasse $56 \%$ de acertos em leitura no DLE, nos pré-testes de unidade, com menos palavras, o seu desempenho foi preciso já no pré-teste; a única exceção foi para a unidade 2 onde obteve $90 \%$ de

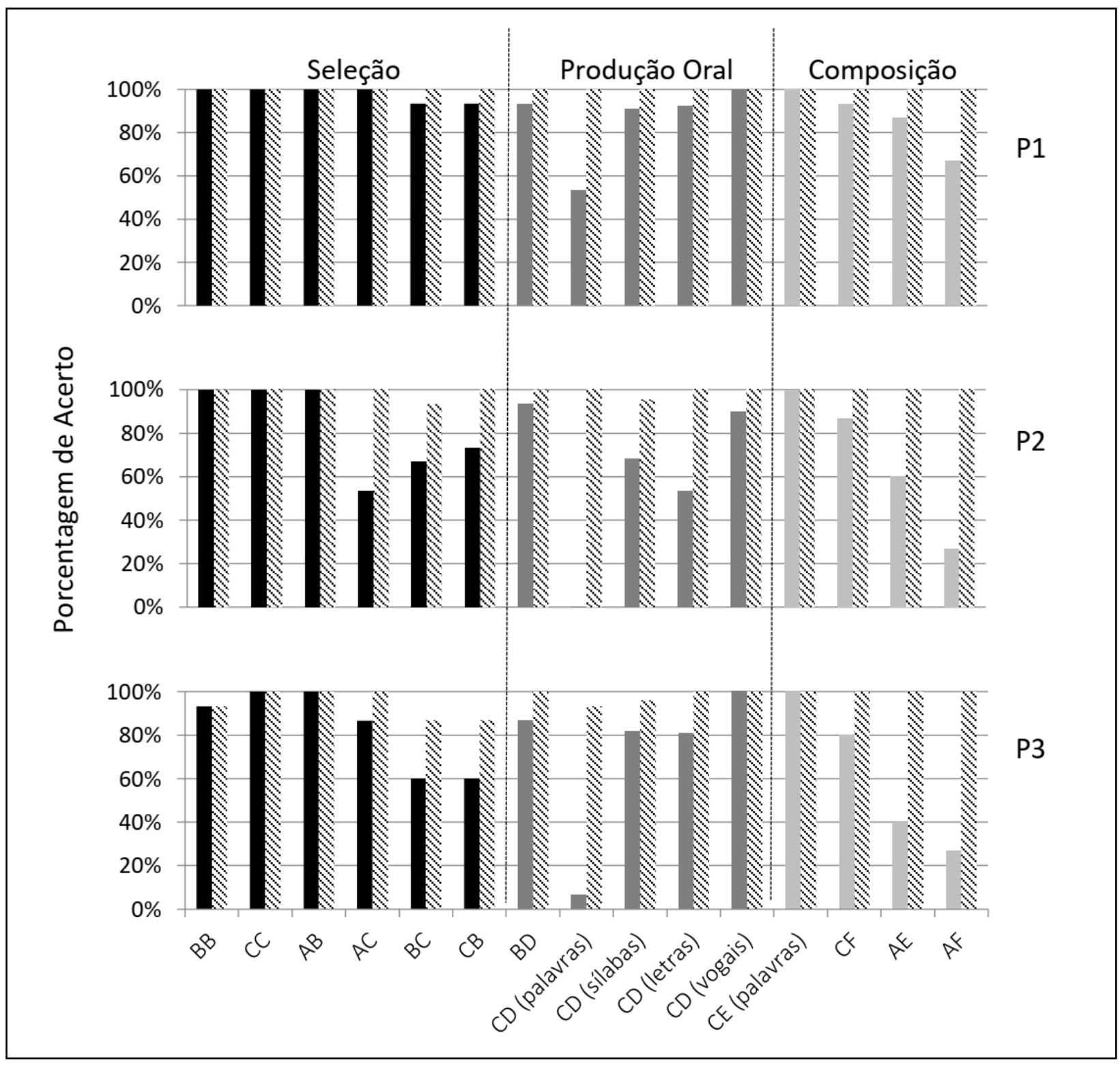

Figura 1. Relações Testadas.

Nota: Desempenho dos participantes nas Tarefas de Seleção, Produção oral e Composição no Diagnóstico de Leitura e Escrita como pré-teste (barras sólidas) e pós-teste (barras hachuradas). As letras representam relações entre estímulos e entre estímulos e respostas. $A, B$ e $C$ representam estímulos, onde $A=$ palavra ditada, $B=$ figura, $C=$ palavra escrita; $D, E$ e $F$ representam respostas, onde $D=$ produção oral, $E=$ construção de respostas via seleção de sílabas e $F=$ manuscrita. 
acertos no pré-teste e chegou à precisão no pós-teste; $\mathrm{P} 2$, que tinha resultados nulos no pré-teste de leitura, apresentou resultados superiores a $60 \%$ nos pós-testes; P3 que já tinha resultados em torno de $50 \%$ de acertos, chegou à precisão nos pós-testes das unidades nas relações baseadas em seleção. Em escrita, considerando os resultados no pré-teste do DLE, os resultados foram de $87 \%$ de acertos para $\mathrm{P} 1,60 \%$ para $\mathrm{P} 2$ e $40 \%$ para $\mathrm{P} 3$; comparando com os pós-testes das Unidades de Ensino, P1 e P3 obtiveram $100 \%$ de acertos e P2 chegou a $90 \%$ na Unidade 2.

\section{Teacher Report Form - TRF}

A Tabela 2 contém os escores do Inventário TRF obtidos antes e depois da intervenção. Para a classificação dos resultados, de acordo com o instrumento, considerou-se que escores igual ou acima de 71 significam que a criança é considerada de nível "clínico" e igual ou abaixo de 66 é considerada como tendo nível "não-clínico". Os escores próximos de 71 (por exemplo, 67-70) são classificados como valores limite entre "clínico" e "não-clínico" (Achenbach \& Rescorla, 2000).

De acordo com a Tabela 2, todos os participantes apresentam nível clínico para problemas de comportamento externalizantes no início do estudo, considerando escore normal até 65, escore limítrofe de 65 a 69 e escore clínico a partir de 70. O participante P1 foi o que apresentou redução importante do escore no TRF (de 80 para 51), saindo de uma classificação considerada clínica para problemas de comportamento externalizantes. Embora P2 tenha diminuído o escore (de 77 para 74), não foi suficiente para que saísse do nível considerado clínico para externalizantes. O participante P3 obteve um aumento no escore (de 71 para 79), permanecendo e agravando a emissão dos problemas de comportamento externalizantes.

Tabela 2. Distribuição dos Escores Obtidos no TRF em Comportamentos Externalizantes no Pré e Pós-Teste.

\begin{tabular}{lcc}
\hline \multirow{2}{*}{ Participantes } & \multicolumn{2}{c}{ Escore total } \\
\cline { 2 - 3 } & Pré-Intervenção & Pós-Intervenção \\
\hline P1 & 80 & 51 \\
P2 & 77 & 74 \\
P3 & 71 & 79 \\
\hline
\end{tabular}

Fonte: Elaboração própria.

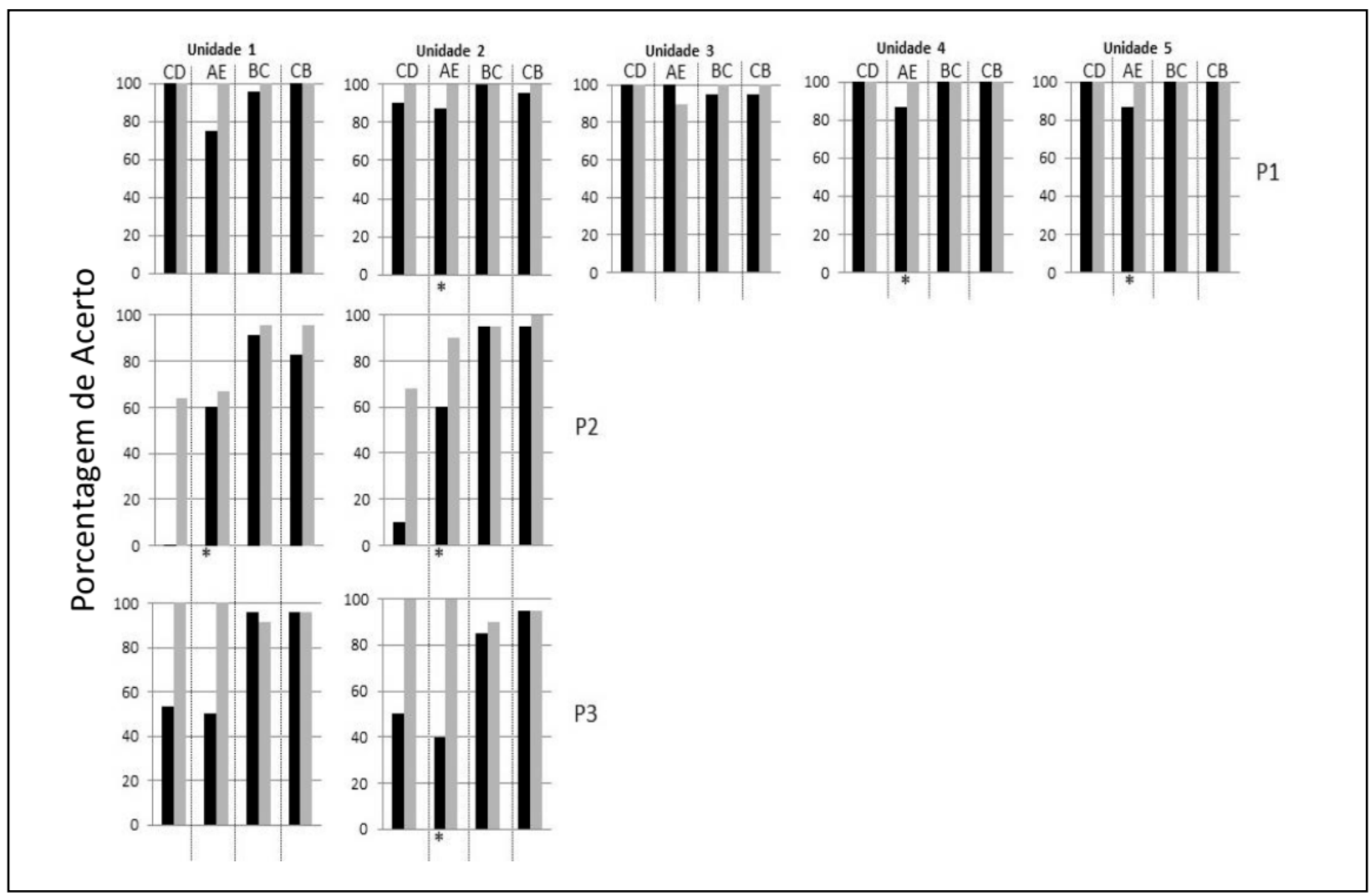

Figura 2. Unidades de Ensino e Relações treinadas.

Nota: Desempenho dos participantes no Pré e Pós testes de Unidades do Programa de Ensino. O asterisco $\left({ }^{*}\right)$ indica que por uma falha na programação, a relação $A E$ não foi testada no Pré teste de Unidade; nesses casos, foi repetida a porcentagem de acertos do DLE. As barras pretas indicam os pré testes e as barras cinzas indicam os pós testes. Siglas das relações: $C D$ - leitura; $A E-$ ditado; $\mathrm{BC}$ - figura - palavra impressa e CB - palavra impressa - figura. 


\section{DISCUSSÃO}

A ocorrência de problemas de comportamentos externalizantes podem dificultar e oferecer barreiras à aprendizagem de outros comportamentos socialmente relevantes, dentre os quais, os acadêmicos (Bandeira et al., 2006). Na literatura, comportamentos externalizantes e repertórios acadêmicos são bastante estudados, porém ou de maneira correlacional ou ainda de maneira independente. Se existem e quais seriam as relações entre esses dois repertórios ainda é assunto pouco explorado (D'abreu \& Marturano, 2010). Esse estudo buscou intervir sobre o comportamento acadêmico de crianças que são marcadamente reconhecidas pelos seus problemas de comportamento externalizantes e que ainda não haviam aprendido os repertórios básicos de leitura e escrita, cursando anos avançados do Ensino Fundamental I. Após a exposição ao programa de ensino (ALEPP) os três participantes demonstraram altas porcentagens de acertos nos pós-testes de avaliação de leitura e escrita (DLE), sobretudo nas principais relações que descrevem leitura e escrita como a medida operacional de compreensão de acordo com o modelo das relações de equivalência ( $B C$ e $C B$ ), a leitura oral de palavras (CD) e a escrita sob ditado (AE).

Esses resultados replicam a literatura com populações bem distintas e público-alvo da Educação Especial (Benitez \& Domeniconi, 2012; de Souza et al., 2019; Gomes \& de Souza, 2016; Lucchesi et al., 2015; Quinteiro, 2015). De maneira geral, os estudos anteriores demonstraram que, com as tarefas de um programa sistematizado de ensino de leitura baseado nas relações de equivalência (de Rose et al., 1989; de Souza et al., 2009) observa-se de maneira eficiente e com poucos erros, a rápida aquisição e refinamento de repertórios acadêmicos em populações diversas. Considerando a generalidade dos resultados para participantes com comportamentos externalizantes o presente estudo estende os efeitos das contingências programadas pelo ALEPP sobre a aprendizagem de leitura e escrita para essa população. Os participantes necessitaram passar por cada passo de ensino apenas uma vez, de forma que as contingências apresentadas pelo ALEPP foram suficientes para manter os participantes engajados na tarefa. Os passos de ensino que constituem as unidades exigem precisão para avançar; o procedimento baseado em exclusão adotado (em que se exclui palavras conhecidas para estabelecer uma relação entre estímulos inédita no repertório) minimizou a ocorrência de erros.

As situações de ensino programadas pelo ALEPP foram suficientes para a manutenção do participante nas tarefas. Avançar na programação após apenas uma exposição às unidades de ensino permite inferir que, de acordo com a análise do comportamento de escolha (Souza \& Coelho, 2019), os participantes preferiram, naquele contexto, realizar as tarefas de leitura e escrita em detrimento da emissão dos comportamentos externalizantes. Os resultados do TRF, discutidos a seguir, corroboram essa interpretação.

Após a exposição ao ALEPP, os três participantes aprenderam relações que envolvem leitura e escrita, chegando ao nível da precisão, aferidos pelo DLE. Independentemente do número de unidades aos quais foram expostos, todos demonstraram altas porcentagens de acertos nos testes de final do programa, sobretudo nas relações que envolvem compreensão (relação entre palavra impressa e figura e vice-versa, BC e $C B$ ), de leitura oral (CD) e de escrita sob ditado (AE). Os resultados dos participantes no DLE final indicaram que os participantes se beneficiaram, e muito, das tarefas de ensino pelo procedimento de CRMTS (Constructed Response Matching-To-Sample), pela sua capacidade de gerar novos repertórios pela aprendizagem da segmentação de palavras que participaram do ensino em unidades menores e da recombinação dessas em novas unidades (de Souza et al., 2009).

A progressão no ensino ocorreu devido às características do ensino individualizado de progressão gradual do conteúdo a ser ensinado e do grau de dificuldade, a partir do estabelecimento de uma linha de base cumulativa, de ensino sem erros e consequências diferenciais para o desempenho. Conforme as contingências programadas pelo ALEPP aumentaram o valor reforçador para a emissão de comportamentos acadêmicos, os participantes passaram a apresentar com frequência maior os comportamentos relacionados à tarefa acadêmica durante as sessões de ensino.

Os resultados no TRF, que é um instrumento baseado no relato do professor, demonstram que os comportamentos externalizantes, reduziram na percepção dessas para P1 e P2, ainda que apenas P1 tenha saído do nível clínico. Esses dados sugerem que, para esses participantes, os problemas de comportamento externalizantes eram competitivos com a aprendizagem de conteúdos acadêmicos. Ao fazer a intervenção sobre os comportamentos acadêmicos, apenas, os participantes desse estudo passaram a emitir mais frequentemente esse comportamento, em detrimento de comportamentos externalizantes assim como no estudo de Guilhardi et al. (1977).

Foram necessárias 66 sessões para que P1 concluísse todas as cinco unidades do ALEPP com êxito e 33 sessões para que P2 e P3 concluíssem duas unidades de ensino. Assim, o programa se mostrou efetivo para ensinar leitura de palavras sem dificuldades ortográficas, em participantes que estavam sendo refratários a esse ensino em condições regulares sem que, contudo, fosse necessário intervir, antes e diretamente, sobre os problemas de comportamento, corroborando, assim como as observações de Guilhardi et al. (1977).

A redução expressiva de problemas de comporta- 
mentos externalizantes não ocorreu para P2 e, para P3, pelo contrário, aumentou. Diante disso, questiona-se se esses participantes tivessem concluído as tarefas de ensino do ALEPP, o escore de problemas de comportamentos externalizantes teria diminuído para níveis não clínicos, como aconteceu com P1.

Uma análise adicional é pertinente. É possível que a manutenção da emissão dos comportamentos acadêmicos em sala de aula em detrimento dos externalizantes possa estar relacionada ao repertório do professor em perceber, selecionar e manter os comportamentos acadêmicos no contexto da sala de aula, favorecendo a generalização desses do contexto individualizado (onde foram aprendidos) para o contexto de aula coletiva onde devem ser mantidos e tornados mais refinados e complexos. Futuros estudos devem controlar melhor a variável repertório do professor no manejo de comportamentos em sala de aula e sua habilidade para reforçar diferencialmente a emissão de comportamentos acadêmicos e externalizantes.

Então, se os professores tivessem recebido um treino de manejo de comportamentos para as situações de sala de aula, teriam sido hábeis em selecionar e manter os comportamentos acadêmicos em uma frequência maior do que os externalizantes? Pesquisas recentes cujo objeto de estudo tem sido o professor como agente responsável pela apresentação das condições de ensino, apontam que as professoras exercem práticas diferenciadas entre os alunos que apresentam problemas de comportamento dos que não apresentam, sendo mais habilidosas nas interações com as crianças sem problemas de comportamento e mais agressivas com as que apresentam problemas de comportamento, o que pode favorecer a emissão de comportamentos inadequados dos alunos (Bolsoni-Silva \& Mariano, 2014).

De maneira complementar, a fim de aprimorar tais práticas educativas, têm sido realizados treinamentos de professores para que esses intervenham de forma mais eficaz diante de problemas de comportamento apresentados pelos alunos, seja com ensino de análise funcional (Ferrari, 2016), softwares para ensino de conceitos de Análise do Comportamento (Fornazari, Kienen, Tadayozzi, Ribeiro, \& Rossetto, 2012), role playing com treinamento de habilidades (Jones, Fremouw, \& Carples, 1977) e treino em equivalência de estímulos (Hayashi, 2007).

Este relato de pesquisa pretendeu contribuir com a discussão entre as áreas da Psicologia e da Educação na abordagem de demandas escolares e educacionais em diferentes níveis de análise e intervenção. No escopo deste trabalho, futuras pesquisas podem verificar a replicabilidade dos resultados obtidos com P1 e sob quais condições uma intervenção sobre comportamentos acadêmicos afetaria a frequência de comportamentos externalizantes, e em que medida a tarefa acadêmica passando a dispor de reforçadores (como a minimização de erros e progressão de acordo com a aprendizagem, como propõe o ALEPP) os problemas de comportamento, por poderem ser concorrentes, tendem a diminuir.

\section{REFERÊNCIAS}

Achenbach, T. M.; Rescorla, L. A. (2000). Manual for the ASEBA School-Age Forms \& Profiles. Burlington, VT: University of Vermont, Research Center for Children, Youth, \& Families.

Bandeira, M.; Rocha, S. S.; Souza, T. M. P.; Del Prette, Z. A. P.; Del Prette, A. (2006). Comportamentos problemáticos em estudantes do ensino fundamental: Características da ocorrência e relação com habilidades sociais e dificuldade de aprendizagem. Estudos de Psicologia (Natal), 11(2), 199-208.

Benitez, P.; Domeniconi, C. (2012). Verbalizações de familiares durante aprendizagem de leitura e escrita por deficientes intelectuais. Estudos de Psicologia (Campinas), 29(4), 553-562.

Bolsoni-Silva, A. T.; Mariano, M. L. (2014). Práticas educativas de professores e comportamentos infantis, na transição ao primeiro ano do Ensino Fundamental. Estudos e Pesquisas em Psicologia, 14(3), 814-833.

Cravo, F. A. M.; Almeida-Verdu, A. C. M. (2018). Avaliação de desempenho escolar após exposição a um programa informatizado de leitura e escrita. Psicologia da Educação, 47, 1-10. https://dx.doi.org/10.5935/2175-3520.20180012

D’Abreu, L. C. F.; Marturano, E. D. (2010). Associação entre comportamentos externalizantes e baixo desempenho escolar: uma revisão de estudos prospectivos e longitudinais. Estudos de Psicologia, 15(1), 43-51.

de Rose, J. C. C.; de Souza, D. G.; Rossito, A. L.; de Rose, T. M. S. (1989). Aquisição de leitura após história de fracasso escolar: Equivalência de estímulos e generalização. Psicologia: Teoria e Pesquisa, 5, 325-346.

de Rose, J. C. (2005). Análise comportamental da aprendizagem de leitura e escrita. Revista Brasileira de Análise do Comportamento, 1, 29-50.

de Souza, D. G.; de Rose, J. C.; Faleiros, T. C.; Bortoloti, R.; Hanna, E. S.; Mcllvane, W. J. (2009). Teaching Generative Reading Via Recombination of Minimal Textual Units: A Legacy of Verbal Behavior to Children in Brazil. International Journal of Psychology and Psychological Therapy, 9, 19-44.

de Souza, D. G.; Caetano, M. S.; Golfeto, R. M.; Postalli, L. M. M.; de Rose, J. C.; Hanna, E. S.;... Rocca, J. Z. (2019). Projeto Alfatech: implementação do currículo ALEPP em escolas públicas da rede municipal de ensino de Santo André - SP (2017-2018). São Carlos: Instituto Nacional de Ciência e Tecnologia sobre Comportamento, Cognição e Ensino. Recuperado de: https://inctecce.com.br/images/ arquivos/00-projeto-alfatech.pdf

Duarte, G. M.; de Rose, J. C. C. (2006). A aprendizagem simbólica em crianças com déficit atencional. Revista Brasileira de Educação Especial, 12(3), 331-350. 
Ferrari, I. P. (2016). Treinamento docente para aplicação de análise funcional baseada em tentativas na avaliação de comportamentos inadequados (Dissertação de Mestrado). Universidade Federal de São Carlos.

Fornazari, S. A.; Kienen, N.; Tadayozzi, D. S.; Ribeiro, G. D.; Rossetto, P. B. (2012). Capacitação de professores em análise do comportamento por meio de programa educativo informatizado. Psicologia da Educação, 35, 24-52.

Gomes, C. G. S.; de Souza, D. G. (2016). Ensino de sílabas simples, leitura combinatória e leitura com compreensão para aprendizes com autismo. Revista Brasileira de Educação Especial, 22(2), 233-252. https://doi.org/10.1590/S141365382216000200007.

Guilhardi, H. J.; Betini, M. E.; Camargo, M. C. S. (1977). Aumento de frequência de respostas acadêmicas para alterar a lentidão e eliminar comportamentos inadequados em um aluno de primeiro grau. Modificação de Comportamento: Pesquisa e Aplicação, 1(2), 1-30.

Guzzo, R. S. L.; Mezzalira, A. S. C.; Moreira, A. P. G.; Tizzei, R. P.; Silva Neto, W. M. F. (2010). Psicologia e Educação no Brasil: uma visão da história e possibilidades nessa relação. Psicologia: Teoria e Pesquisa, 26(spe), 131141. https://doi.org/10.1590/S0102-37722010000500012

Hanley, G. P.; Iwatta, B. A.; McCord, B. E. (2003). Functional analysis of problem behavior: a review. Journal of Applied Behavior Analysis, 36(2): 147-185.

Harris, V. W.; Sherman, J. A. (1974). Homework assignments, consequences, and classroom performance in social studies and mathematics. Journal of Applied Behavior Analysis, 7, 505-519.

Hayashi, E. A. P. (2007). A metodologia da equivalência de estímulos como recurso pedagógico para o professor do contraturno (Tese de Doutorado). Universidade Estadual Paulista Júlio de Mesquita Filho, Marília - SP.

Jones, F. H.; Fremouw, W.; Carples, S. (1977). Pyramid training of elementary school teachers to use a classroom management skill package. Journal of Applied Behavior
Analysis, 10, 239-253.

Lucchesi, F. D. M.; Almeida-Verdu, A. C. M.; Buffa, M. J. M. B.; Bevilacqua, M. C. (2015). Efeitos de um Programa de Ensino de Leitura sobre a Inteligibilidade da Fala de Crianças Usuárias de Implante Coclear. Psicologia Reflexão e Crítica, 28(3), 500-510. https://doi.org/10.1590/16787153.201528309 .

Paula, F, S.; Tfouni, L. V. (2009). A persistência do fracasso escolar: desigualdade e ideologia. Revista Brasileira de Orientação Profissional, 10(2), 117-127.

Quinteiro, R. S. (2014). Aquisição de discriminações auditivotáteis e emergência de leitura recombinativa em Braille (Tese de Doutorado). Universidade Federal de São Carlos.

Reis, T. S.; de Souza, D. G.; de Rose, J. C. (2009). Avaliação de um programa para o ensino de leitura e escrita. Estudos em Avaliação Educacional, 20, 425-450.

Rosa Filho, A. B.; de Rose, J. C. C.; de Souza, D. G.; Hanna, E. S.; Fonseca, M. L. (1998). Aprendendo a ler e a escrever em pequenos passos. Software para pesquisa.

Santos, S. L. R.; Almeida-Verdu, A. C. (2012). Leitura em uma criança surda após equivalência de estímulos. Psicologia em Revista, 18(2), 209-226.

Sidman, M. (2000). Equivalence relations and the reinforcement contingency. Journal of the Experimental Analysis of Behavior, 74, 127-146.

Sidman, M.; Tailby, W. (1982). Conditional discrimination vs. matching to sample: An expansion of the testing paradigm. Journal of the Experimental Analysis of Behavior, 37, 5-22.

Sousa, J. B.; Coelho, C. (2019). Análise comportamental de padrões parentais a partir da noção de autocontrole e impulsividade. Revista Estudos - Zootecnia, 46, 65-80. http://dx.doi.org/10.18224/evs.v46i1.7243

Zaine, I.; Domeniconi, C.; de Rose, J. C. (2014). Simple and Conditional Discrimination and Specific Reinforcement in Teaching Reading: An Intervention Package. The Analysis of Verbal Behavior, 30(2), 193-204.

As autoras são membros do Instituto Nacional de Ciência e Tecnologia sobre Comportamento, Cognição e Ensino (INCT-ECCE), financiado pela FAPESP ((Processo \#2008/57705-8) e pelo CNPq (Processo \#573972/2008-7). Este artigo é resultado da dissertação de mestrado, título: Efeitos do ensino sistemático e informatizado sobre comportamentos externalizantes concorrentes à aprendizagem de leitura e escrita, orientado por Ana Claudia Moreira Almeida -Verdu, concluída em 2014, financiada pela FAPESP, processo 2012/23526-5. 